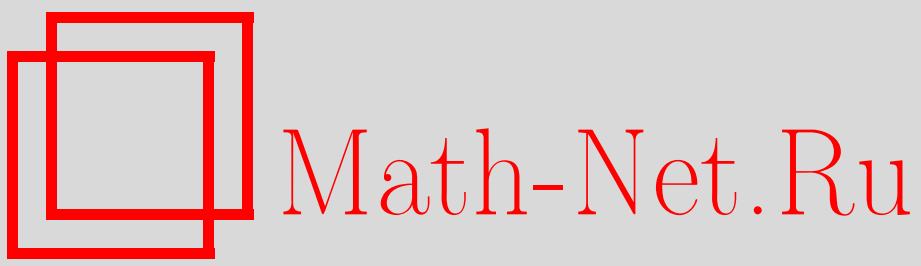

И. Купш, О. Г. Смолянов, О моделях симметричной алгебры Фока, Матем. заметки, 1996, том 60, выпуск 6, 939-942

DOI: https://doi.org/10.4213/mzm1914

Использование Общероссийского математического портала MathNet.Ru подразумевает, что вы прочитали и согласны с пользовательским соглашением

http://www. mathnet.ru/rus/agreement

Параметры загрузки:

IP : 3.89 .197 .203

26 апреля 2023 г., 14:00:58

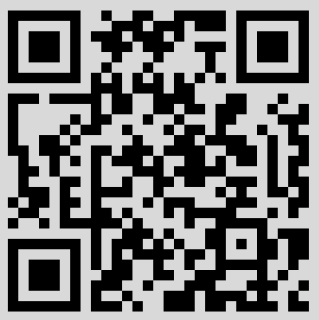




\section{О МОДЕЛЯХ СИММЕТРИЧНОЙ АЛГЕБРЫ ФОКА}

\section{И. Купш, О.Г. Смолянов}

Описываются две реализации суперпространства, соответствующего рассмотренной в [1] (см. также [2]) суперсимметричной алгебре Фока, порождаемой гильбертовым пространством $E$. В обеих реализациях важную роль играет свободный модуль $E_{\Lambda}$ над некоторой алгеброй $\Lambda$, порождаемый $E$ (он и назьвается суперпространством, соответствующим $E$ ). При этом в одной из рассматриваемых реализаций используется (супер) пространство (некоторых) функций на $E_{\Lambda}$, принимающих значения в $\Lambda$; в другой реализации используется прямая сумма симметризованных (конечных) тензорных произведений суперпространства. Обе эти реализации аналогичны (восходящим к Фоку, Фридрихсу и Сигалу) представлениям ("бозонного") пространства Фока. Пространство $E_{\Lambda}$ наделяется так называемой гауссовской супермерой [3], [4], что позволяет автоматически получать аналоги гауссовских комбинаторных соотношений. В то же время, использование пространства функций, принимающих значения в супералгебре, позволяет связать умножение в супералгебре Фока с поточечным умножением этих функций. Кроме того, в заметке описываются некоторые бесконечномерные (супер) дифференциальные операторы, являющиеся гамиль тонианами (бесконечномерных) супераналогов квантового ангармонического осциллятора, и рассматриваются связи между их различными представлениями. В частности, вводится оператор, который можно назвать производящим оператором процесса Орнштейна-Уленбека в суперпространстве, и его обобщения. Результаты первой части заметки можно распространить на случай, когда вместо гильбертова пространства используется ядерное пространство Фреше.

1. Обозначения, терминология и предварительные сведения. Термины и обозначения из [5] используются без пояснений. Все топологические пространства предполагаются отделимыми. Векторное пространство называется $Z_{2}$-градуированным, если оно является прямой суммой двух своих векторных подпространств, индексированных символами 0 и 1 и называемых, как и их элементы, соответственно четным и нечетным; если $E=E_{0} \oplus E_{1}$ $Z_{2}$-градуированное векторное пространство, то через $p_{E}$ обозначается функция на $\left(E_{0} \cup E_{1}\right) \backslash\{0\}$, равная нулю на элементах из $E_{0}$ и единице - на элементах из $E_{1}$ (для всякого $a \in\left(E_{0} \cup E_{1}\right) \backslash\{0\}$ число $p_{E}(a)$ называется четностью $a$ ). Супералгебра (над полем $K$ ) - это $Z_{2}$-градуированное векторное пространство $\Lambda=\Lambda_{0} \oplus \Lambda_{1}$ над полем $K$, наделенное ассоциативным умножением $(a, b) \mapsto a b$, обладающим следующими свойствами: если $a, b \in\left(E_{0} \cup E_{1}\right) \backslash\{0\}$, то $a, b \in E_{0} \cup E_{1}$, причем если $a b \neq 0$, то $p_{E}(a b)=\left|p_{E}(a)-p_{E}(b)\right|$. Супералгебра назьвается $(c y$ nер) коммутативной, если при тех же предположениях $a b=(-1)^{p_{E}(a) p_{E}(b)} b a$. Далее предполагается, что поле скаляров - это поле комплексных или поле вещественных чисел. Банахова (гильбертова) супералгебра - это супералгебра, на которой определена норма (гильертова норма), превращающая ее в банахову алгебру. Всюду далее $\Lambda=\Lambda_{0} \oplus \Lambda_{1}$ - коммутативная гильбертова супералгебра над полем вещественных чисел, $\Lambda^{c}$ - ее комплексификация; предполагается, что $\left\{\lambda \in \Lambda: \forall \eta \in \Lambda_{1} \lambda_{\eta}=0\right\}=\{0\}$ (при этом каноническое вложение $\Lambda$ в пространство всех $\Lambda$-линейных отображений $\Lambda$ в $\Lambda$ оказывается инъективньм). Типичный пример такой супералгебры - это пополнение грассмановой алгебры 
со счетньм числом образующих по подходящей гильбертовой норме. Если $H$ -

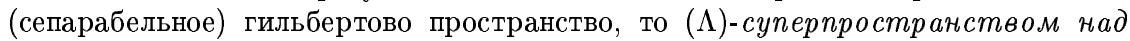
$H$ назьвается $Z_{2}$-градуированный $\Lambda$-модуль

$$
H_{\Lambda}=\left(H_{\Lambda}\right)_{0} \oplus\left(H_{\Lambda}\right)_{1}=\left(\Lambda_{0} \otimes H\right) \oplus\left(\Lambda_{1} \otimes H\right)(=\Lambda \otimes H) .
$$

Здесь символ ' $\otimes$ ' обозначает пополнение предгильбертова пространства, представляющего собой тензорное произведение соответствующих гильбертовых пространств, наделенное (единственной) гильбертовой нормой, являющейся произведением (гильбертовых) норм перемножаемых пространств (это частный случай определения из [3], см. также [6]). Супералгебра $\Lambda$ далее отождествляется с суперпространством над $\mathbb{R}^{1}$. Если $E$ и $G$ - два гильбертовых пространства, то символом $\mathscr{L}_{2}\left(E_{\Lambda}, G_{\Lambda}\right)$ обозначается $\Lambda$-модуль всех $\Lambda$-гомоморфизмов $E_{\Lambda}$ в $G_{\Lambda}$, являюшихся $\Lambda$-линейными продолжениями отображений Гильберта-Шмидта пространства $E$ в пространство $G$; таким образом, $\mathscr{L}_{2}\left(E_{\Lambda}, G_{\Lambda}\right)$ изоморфно $\Lambda$-модулю $\Lambda \otimes \mathscr{L}_{2}(E, G)$ (где $\mathscr{L}_{2}(E, G)$ - гильбертово пространство всех отображений Гильберта-Шмидта из $E$ в $G)$, и мы предполагаем, что $\mathscr{L}_{2}\left(E_{\Lambda}, G_{\Lambda}\right)$ наделено структурой гильбертова пространства, определяемой этим изоморфизмом (таким образом, $\mathscr{L}_{2}\left(E_{\Lambda}, G_{\Lambda}\right)$ является некоммутативной гильбертовой супералгеброй). Отображение $f: E_{\Lambda} \rightarrow G_{\Lambda}$ называется (супер) дифферениируемым в точке $x \in E_{\Lambda}$, если оно дифференцируемо в этой точке по Фреше как отображение гильбертова пространства $E_{\Lambda}$ в гильбертово пространство $G_{\Lambda}$, причем его производная Фреше $f^{\prime}(x)$ в точке $x$ (называемая в этом случае также суперпроизводной) является элементом пространства $\mathscr{L}_{2}\left(E_{\Lambda}, G_{\Lambda}\right)$. Кратная дифференцируемость, производные высших порядков и аналитичность отображения определяются естественным образом. Отметим еще, что если отображение (открытой части) суперпространства $E_{\Lambda}$ в $\Lambda \quad n$ раз дифференцируемо в точке $x \in E_{\Lambda}$, то сужение производной $f^{\prime}(x)$ на $\left(E_{\Lambda}\right)_{1}$ можно отождествить с элементом тензорного произведения $\Lambda \otimes \bigwedge_{n} E$, где символ $\bigwedge_{n} E$ обозначает гилббертово пространство, представляющее собой замкнутое векторное подпространство пространства $E \otimes E \otimes E \otimes \cdots \otimes E(n$ раз), порожденное (алгебраическим) внешним произведением $n$ экземпляров пространства $E$ [3], [4].

2. Супермера и суперскалярное произведение. Пусть $H$ - (сепарабельное) гильбертово пространство. Суперскалярным произведением в $H_{\Lambda}$ называется непрерьвное $\Lambda$-билинейное отображение $b: H_{\Lambda} \times H_{\Lambda} \rightarrow \Lambda$, обладающее следуюшими свойствами: сужения $b$ на $\left(H_{\Lambda}\right)_{0} \times\left(H_{\Lambda}\right)_{1}$ и на $\left(H_{\Lambda}\right)_{1} \times\left(H_{\Lambda}\right)_{0}$ равны нулю; сужение $b$ на $\left(H_{\Lambda}\right)_{1} \times\left(H_{\Lambda}\right)_{1}$ совпадает с сужением на это множество $\Lambda$-билинейного продолжения на $H_{\Lambda} \times H_{\Lambda}$ некоторого невырожденного антисимметричного билинейного функционала на $H$, а сужение $b$ на $\left(H_{\Lambda}\right)_{0} \times\left(H_{\Lambda}\right)_{0}-\mathrm{c}$ сужением на это множество $\Lambda$-билинейного продолжения на $H_{\Lambda} \times H_{\Lambda}$ (обычного) скалярного произведения на $H$. Пусть еще $\mathscr{F}(H)-\left(Z_{2}\right.$-градуированное $)$ пространство всех аналитических отображений $f$ суперпространства $H_{\Lambda}$ в $\Lambda^{c}$ таких, что если

$$
(D f)(x)=\left(f(x), f^{\prime}(x) \uparrow\left(H_{\Lambda}\right)_{1}, \ldots, f^{(n)}(x) \uparrow\left(\left(\bigwedge_{n} H\right)_{\Lambda}\right)_{1}, \ldots\right)
$$

то $(D f)(x) \in \Lambda^{c} \otimes(\wedge H)$, где $\wedge H$ - это гильбертова сумма семейства $\left\{\bigwedge_{n} H\right\}$ гильбертовых пространств. Отметим, что каждая функция $f \in \mathscr{F}(H)$ однозначно определяется своим сужением на канонический образ $H_{0}$ пространства $H$ в $H_{\Lambda}$. 
ОПРЕДЕЛЕНИЕ 1 (см. [3]). Супермерой на $H_{\Lambda}$ называется борелевская счетно аддитивная мера на $H_{0}$, принимающая значения в $\Lambda^{c} \otimes \wedge H$.

Если $\nu$ - супермера на $H_{\Lambda}$, то интеграл функции $f \in \mathscr{F}(H)$ по супермере $\nu$, обозначаемый символом $\int f d \nu$, определяется равенством $\int f d \nu=\int_{H}((D f)(x), \nu(d x))$ здесь и далее символ $(\cdot, \cdot)$ обозначает подходящее продолжение скалярного произведения в $H$. Преобразование Фурье супермеры $\nu$ на $H_{\Lambda}-$ это функция $F_{\nu}$ на $H_{\Lambda}$, определяемая так: $F_{\nu}(x)=\int \exp i(x, z) \nu(d z)$. Супермера $\nu$ на суперпространстве $H_{\Lambda}$ называется гауссовской, если на $H_{\Lambda}$ существует такое (называемое коррелячионным функиионалом меры $\nu$ ) суперскалярное произведение $\langle\cdot, \cdot\rangle$, что $F_{\nu}(x)=\exp (-\langle x, x\rangle / 2)$, где $x \in H_{\Lambda}$. Супермера $\nu$ на суперпространстве $H_{\Lambda}$ назьвается (супер) дифферениируемой в направлении $h \in H_{\Lambda}$, если существует такое $\nu$-интегрируемое отображение $\beta(h, \cdot): H_{\Lambda} \rightarrow \Lambda$ (назьваемое логари мической производной супермеры $\nu$ в направлении $h \in H_{\Lambda}$ ), что для всякой функции $\varphi \in \mathscr{F}(H)$

$$
\int \varphi^{\prime}(x) h d \nu=-\int \varphi \beta(h, x) d \nu,
$$

когда оба интеграла существуют.

ПРЕДЛОЖЕНИЕ 1. Пусть $\nu$ - гауссовская супермера на суперпространстве $H_{\Lambda}$, причем существует такое отобрахсение $B: H_{\Lambda} \rightarrow H_{\Lambda}$ (назьваемое коррелячионным оператором супермеры $\nu)$, что $\langle x, z\rangle=(B x, z)$

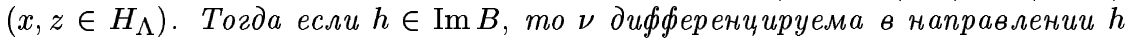
$u \beta(h, x)=-\left(B^{-1} h, x\right)$.

3. Модели суперпространств Фока. Пусть $H_{s}$ - симметричное и $H_{a}-$ антисимметричное пространства Фока, соответствующие (гильбертову) пространству $H$; в этих пространствах Фока, помимо структуры гильбертова пространства, имеются также структуры алгебры $\left(H_{s}\right.$ - это гильбертова сумма симметризованных гильбертовых тензорных произведений комплексификаций $H^{c}$ пространства $H$ ).

Пусть символы ' $\mathrm{V}$ ' и ' $\wedge$ ' обозначают операции умножения соответственно в $H_{s}$ и в $H_{a}$, и пусть $E=H_{a} \otimes H_{s}$ и для $x_{j} \otimes z_{j} \in E(j=1,2)$

$$
\left(x_{1} \otimes z_{1}\right) \circ\left(x_{2} \otimes z_{2}\right)=\left(x_{1} \wedge x_{2}\right) \otimes\left(z_{1} \vee z_{2}\right)
$$

Тогда $E$ - это “суперсимметричная алгебра Фока" ([1], [2]).

Пусть теперь $\sum H_{\Lambda}^{c}$ - гильбертова сумма суперсимметризованных тензорных произведений $H_{\Lambda}^{c} \otimes \cdots \otimes H_{\Lambda}^{c}$. Обозначим, далее, через $\mathscr{F}_{\nu}(H)$ пополнение предгильбертова пространства, получаемого путем наделения подходящего подпространства (супер) пространства $\mathscr{F}(H)$ гильбертовой нормой $\|\cdot\|$, определяемой равенством $\|f\|^{2}=\int_{H}\|(f(x) \overline{f(x)}, d \nu)\|_{\Lambda}$, где $\nu$ - некоторая гауссовская супермера на $H_{\Lambda}$ и $\|\cdot\|_{\Lambda}$ - норма в $\Lambda$.

ТЕОРема 1. Существует (естественный) изоморфизм (супер) алгебрь $E_{\Lambda}$ на супералгебру $\sum H_{\Lambda}^{c}$, индущирующий представление (супер) алгебрь E в $\sum H_{\Lambda}^{c}$. Кроме того, каждое из изоморфных суперпространств $E_{\Lambda} u$ $\sum H_{\Lambda}^{c}$ изоморфно суперпространству $\mathscr{F}_{\nu}$ (соответствующий изоморфизм является продолхением изоморфизма Сигала).

ЗАмечАниЕ 1. Аналог теоремы 1 справедлив и для тензорного произведения $H_{s} \otimes K_{a}$, где $H, K-$ два (различных) гильбертовых пространства. 
4. Супердифференциальные операторы. Линейные супердифференциалные операторы определяются как псевдодифференциальные операторы с подходящими символами. Мы рассмотрим здесь только символы $\mathscr{H}(q, p)$ вида $\mathscr{H}(q, r)=\frac{1}{2}\langle p, p\rangle+V(q)$, где $(q, p) \in Q \times P$, причем $Q$ и $P$ - два экземпляра суперпространства над гильбертовым пространством $H$, наделенного суперскалярным произведением $\langle\cdot, \cdot\rangle$. Пусть $\widehat{\mathscr{H}}-$ псевдодифференциальный оператор в $H_{\Lambda}$ с символом и $\nu$ - супермера на $H_{\Lambda}$, логарифмическая производная которой удовлетворяет соотношению $V(q)=\beta^{\prime}(\cdot, q)+\operatorname{str} \beta(\cdot, q) \otimes \beta(\cdot, q)$ для каждого $q \in Q$.

Teорема 2. Если $\widehat{\mathscr{H}}_{1}$ - линейное отображсение $\mathscr{F}_{\nu}(Q)$ в $\mathscr{F}(Q)$, определяемое равенством $\widehat{\mathscr{H}}(\varphi, \nu)=\left(\widehat{\mathscr{H}}_{1} \varphi\right) \cdot \nu$, то для каждого $\varphi \in \mathscr{F}_{\nu}(Q)$

$$
\widehat{\mathscr{H}}_{1} \varphi(\cdot)-\frac{1}{2} \operatorname{str} \varphi^{\prime}(\cdot)-\operatorname{str} \varphi^{\prime} \otimes \beta(\cdot, \cdot) .
$$

ЗАмечание 2 . Пусть $V(\cdot)=\langle\cdot, \cdot\rangle$. Тогда оператор $\widehat{\mathscr{H}}_{1}$ естественно считать супераналогом (бесконечномерной версии) классического оператора ОрнштейнаУленбека.

Университет Кайзершлаутер

Поступило

Московский государственный университет

16.05 .95

им. М. В. Ломоносова

\section{СПИСОК ЦИТИРОВАННОЙ ЛИТЕРАТУРЫ}

1. Kupsh J. // Rev. Math. Phys. 1990. V. 2. № 4. P. 457-477. 2. Haba Y., Kupsh J. // Forschr. Phys. 1995. V. 45. №1. Р. 41-66. 3. Смолянов О. Г., Шавгулидзе Е.Т. // Докл. АН СССР. 1988. Т. 299. №4. С. 476-482. 4. Смолянов О.Г., Шавгулидзе Е.Т. // Докл. АН СССР. 1989. Т. 309. №3. С. 545-550. 5. Шефер Х. Топологические векторные пространства. М.: Мир, 1971. 6. Владимиров В. С., Волович И. В. // Докл. АН СССР. 1985. T. 285. №6. C. 1042-1044. 